\title{
Home remedies for Pediculus humanus capitis infection among schoolchildren
}

\section{Hiro Mohammed Obaid}

\author{
Kirkuk Technical College, Medical Laboratory Techniques Department, Kirkuk, Iraq \\ Corresponding author: Dr. Hiro Mohammed Obaid, E-mail: dr.obaidhm13@gmail.com
}

\begin{abstract}
Background: Head lice (HL) is still signifying problem, its incidence is greater in crowded places like schools and day cares. Material and Methods: A total of 1791 school children, from five schools (three elementary and two intermediate) in Kirkuk province, Iraq, were eligible for screening of head lice. The heads were examined carefully by naked eye or with assistance of magnifying lens. After detecting the infected heads, natural and chemical products were used for treating the infection. Results: 255 samples were found to be positive for head lice, with rate of $14.2 \%$. Females were significantly more infected than males. The most age group which was infected in both genders was 7-8 and 9-10 years old. parents occupation or the educational status of the infected children, were not associated with the incidence of the infection. The effect of the natural plant oils were intensified when used with head cap. The most effective plant oil was olive and anise followed by thyme and sesame they were effective at 48 hours after usage. Very little effect had resulted with garlic oil. Petrol was effective in killing the lice with or without head cap. The anti-lice shampoos were effective after repeating treatment for four to five days. The ordinary hair shampoos were effective at killing the lice only when used with head cap. Olive oil and sesame are effective for head lice treating, but the commercial normal hair shampoos are better for completely removing the infection, if used with hair cap. Conclusion: The recommendation is that, the ordinary hair shampoos can be used instated of natural oils or anti-lice shampoos that contain insecticides and may have side effects.
\end{abstract}

Key words: Head lice; Home remedies; School children

\section{INTRODUCTION}

Head lice belong to Anoplura (sucking lice), it's an obligate ectoparasite. Human being is the only known host for the head, body and crab lice, however, there is evidence that crab lice can infect gorilla [1]. Head lice cannot fly or jump. Therefore, it transmit from one to another by direct contact, or by using hair combs, brushes, hats and bedding of infected person [2]. HL sucks human blood for nourishment causing head itching and discomfort. The infection may also cause anemia particularly in individuals with low hemoglobin level. A greater importance is that, their saliva may led to allergy, psychological effect and absence from school $[1,3]$. Factors as crowd and poverty may enhance the infection [4]. Despite the huge number of chemical components used for treating the infection, HL is still prevalent worldwide [5]. In Iraq and Arab countries the parasite rate is fluctuating. In Kirkuk city, in 16 primary schools, of 1130 pupils (828 girls, 302 boys) examined for HL. A rate of $20 \%$ was positive [3]. From eight elementary schools in Baghdad, 540 boys and girls, the total rate of head lice was $13.5 \%$ [6]. The head lice prevalence among 5150 Jeddah city Saudian girls were $1.26 \%$ [7]. The overall prevalence of pediculosis was $23.32 \%$, among 747 schoolchildren from 12 primary school of Ladkrabang district, Bangkok, Thailand [8].

Preschool and elementary school aged children were most common infested with head lice in the United States [9]. The prevalence was $43 \%$ in a slum and $28 \%$ in a fishing village in Brazil [4]. Many chemical products were investigated for their effects on HL, in United States the most studied pediculicide was Permethrin, the least toxic to human [10]. In 2009, $5 \%$ benzyl alcohol was inspected as a treatment for HL

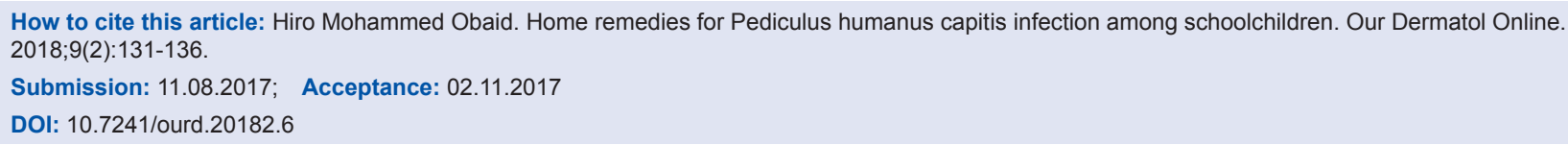


in children older than 6 months [11]. Three head lice treatment options: malathion, pyrethrins and piperonyl butoxide was conducted and studied in children who had severe head lice infestations in Queensland primary schools, [12]. But no or very little effects were recorded in all mentioned studies. Anise, ylang-ylang, coconut oils and isopropyl alcohol was found to be at least as effective as the permethrin [13]. Because of the urgency for new therapies, due to resistance phenomenon appeared in HL against chemical product, the absence of eradicative treatment and HLincreases in human population, the aim of the present study was to detect the rate of head lice in Kirkuk province and practice some natural and chemical products HL.

\section{MATERIALS AND METHODS}

\section{Methods}

Population Study: From September 2015 to April 2016, a total 1791 school children were screened for head lice presence. The children were from five schools (three elementary and two intermediate)in Kirkuk province, Iraq. Data including gender, age, head or body itching, parents occupation, economic and educational status of all infected children were recorded.

Head screening and sample collection: The head of all children were examined carefully by naked eye or with assistance of magnifying lens. After detection of insect stages (nit, nymph, adult), they were transferred into disposable cup with tide lid. The specimens were bring to the laboratory for microscopic examining.

Treatment groups: 190 person of heavy or moderate infected were agreed to be chosen for treatment experiments. they were divided in to two main groups (120 infected person for natural products and for 70 person for chemicals). From each of the two groups a number of 10 infected person were used for each product (two person for each time used).

Treatment experiments: Different natural and chemical components were used in lice treating. The natural products, composed of plant oil (olive, thyme, anise, sesame, garlic and apple vinegar), these was obtained from local markets. Chemicals were petrol, anti-lice shampoos (Sali, lice therapy and lycid). In addition to ordinary or traditional hair shampoos. All products except anti-lice shampoos were tested with or without using a plastic head cap. The cap when used, was completely covered the head along with the ears and well tide retaining the air form reaching the head. The natural components were left on the infected head to different times $(2,8,12,24,48$ hours) while the chemical components was used for 1-5 days (anti-lice shampoos were used according to instructions on their bottles). A towel was put around the plastic cap, to prevent the leak out. After applying a massive amount of plant natural oils or shampoos on the infected head. They were mixed thoroughly with all parts of the hair. After each time or day of treating, the heads were examined periodically for the presence or absence of the lice stages. Afterword (two to eight weeks) the treated heads were reviewed for the recurrence of the infection. Along with hair treatment the families of treated groups were asked to expose the clothing and bedding to heat or to sun for two to five days.

2-4-Statistical Analysis: T test and $\chi^{2}$ (chi-square) test in style of independent and in style of homogeneous were used manually. The significant level used was $\mathrm{P}<0.01$ or 0.05 .

\section{RESULTS}

This study had designed to find out the prevalence of head lice in children of Kirkuk city. All stages of the HL were seen and magnified microscopically, Figs. 1-3 denotes these stages.

A significant differences of head lice infection was noted between female and male, Table 1 . The rate of infection was higher in female (23.6\%) comparing with that in male $(3.2 \%)$. The overall frequency rate of HL among children of Kirkuk province was $14.2 \%$.

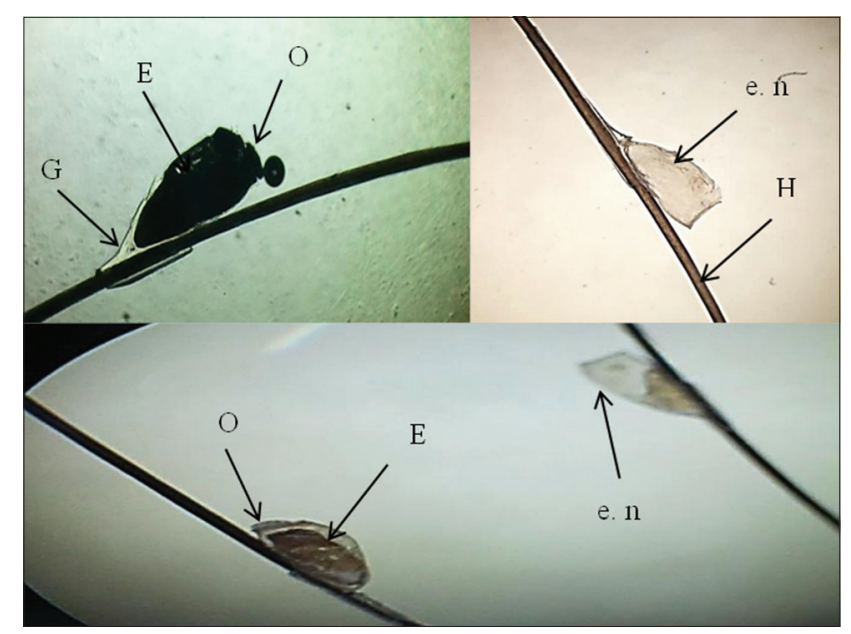

Figure 1: The embryo containing or empty nits of head Lice. ( $E=$ embryo, $\mathrm{O}=$ opercula, $\mathrm{G}=$ glue used for egg attaching with hair, e. $n=$ empty nit, $\mathrm{H}=$ hair shaft). 


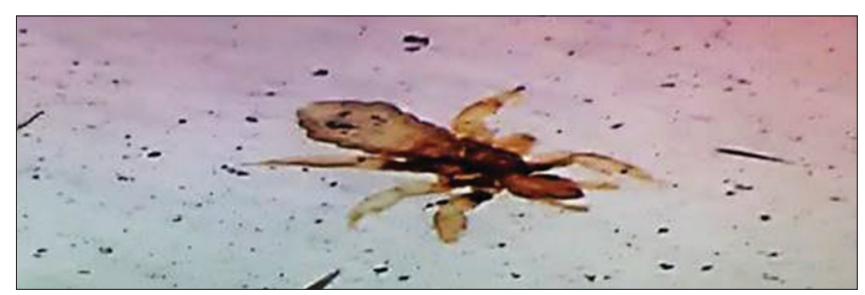

Figure 2: The nymph stage of head lice.

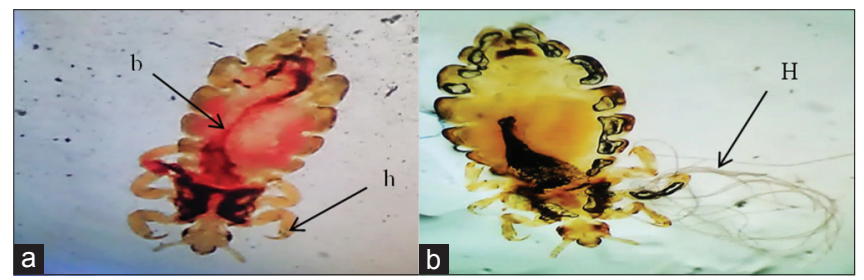

Figure 3: The adult stages of head lice (a) a blood sucked adult, (b) none blood sucked adult) $\mathrm{b}=$ blood, $\mathrm{H}=$ hairs, $\mathrm{h}=$ hanging claw on the leg.

The result in Table 2 indicates, that there were significant differences in HL infection between age groups. The most age group which was infected in both females and males were 7-8 and 9-10 years old. With a rate of $41,32.8 \%$ for females and a rate of $13,7 \%$ for males in each age group respectively.

The recurrence of HL infection is illustrated in Table 3. The result was significant for those had continuous infection comparing with children infected for first or second time. In female the maximum infection rate $(65 \%)$ was for continuous infection, while in male was for those infected for first time with rate of $73.1 \%$.

HL infection had well related with head or body itching, Table 4. A rate of $44.9 \%$ of infected persons had suffered from head itching and a rate of $15.3 \%$ had suffered from both head and body itching, whereas $40 \%$ of them had no head or body itching.

As indicated in Table 5, the rate of heavy infected persons were significantly higher $(64.3 \%)$ than moderately $(20.8 \%)$ or lightly $(14.9 \%)$ infected.

The parents occupation or educational status of the infected children, were significantly not associated with the incidence of the infection. Both employed or un employed parents or parents at different levels of educational status had infested child with nearly equal rates.

All families with different levels of economic status had infested child. But the greatest percentage was for intermediate level with rate of $66.5 \%$, followed by
Table 1: Head lice frequency among school children

\begin{tabular}{lccr}
\hline Gender & Total no. examined & +ve (\%) & -ve (\%) \\
\hline Female & 971 & $229(23.6)$ & $742(76.4)$ \\
Male & 820 & $26(3.2)$ & $794(96.8)$ \\
Total & 1791 & $255(14.2)$ & $1536(85.8)$ \\
T value & Evaluated T value=29.5, T value of $\mathrm{P}<0.05=6.31\left(\right.$ significant $\left.^{* *}\right)$ \\
\hline
\end{tabular}

Table 2: Head lice frequency according to pupils age

\begin{tabular}{lcc}
\hline Age group in years & \multicolumn{2}{c}{ Gender } \\
\cline { 2 - 3 } & Female & Male \\
\cline { 2 - 3 } & +ve (\%) & +ve (\%) \\
\hline $7-8$ & $94(41.0)$ & $13(50)$ \\
$9-10$ & $75(32.8)$ & $7(26.9)$ \\
$11-12$ & $42(18.3)$ & $6(23.0)$ \\
$13-14$ & $18(7.9)$ & $0(0)$ \\
Total & 229 & 26 \\
T value & Evaluated T value=2.55, T value of \\
& P<0.05=2.35 (significant) \\
\hline
\end{tabular}

Table 3: Head lice frequency according to head or body itching

\begin{tabular}{lccc}
\hline Gender & $\begin{array}{c}\text { No. had head } \\
\text { itch's (\%) }\end{array}$ & $\begin{array}{c}\text { No. had head } \\
\text { and body } \\
\text { itch's }(\%)\end{array}$ & $\begin{array}{c}\text { No. had no } \\
\text { head or body } \\
\text { itch's }(\%)\end{array}$ \\
\hline Female & $94(41.0)$ & $37(16.1)$ & $98(42.8)$ \\
Male & $20(76.9)$ & $2(7.7)$ & $4(15.4)$ \\
Total & $114(44.9)$ & $39(15.3)$ & $102(40)$ \\
$\chi^{2}$ value & Evaluated $\chi^{2}$ value $=12.21, \chi^{2}$ value of P<0.01=9.21 (significant) \\
\hline
\end{tabular}

Table 4: Some natural oils effect on head lice viability

\begin{tabular}{|c|c|c|c|c|c|}
\hline \multirow[t]{2}{*}{ Natural oils } & \multicolumn{5}{|c|}{ Time in hours } \\
\hline & 2 & 8 & 12 & 24 & 48 \\
\hline \multicolumn{6}{|l|}{ Olive } \\
\hline W & A & A & A & A & A \\
\hline Wc & A & A & $A+D$ & $A+D$ & D \\
\hline \multicolumn{6}{|l|}{ Thyme } \\
\hline W & A & A & A & A & A \\
\hline Wc & A & A & A & $A+D$ & D \\
\hline \multicolumn{6}{|l|}{ Anise } \\
\hline w & A & A & A & A & A \\
\hline Wc & A & A & $A+D$ & $D$ & D \\
\hline \multicolumn{6}{|l|}{ Sesame } \\
\hline w & A & A & A & A & A \\
\hline Wc & A & A & $A+D$ & $A+D$ & D \\
\hline \multicolumn{6}{|l|}{ Garlic } \\
\hline W & A & A & A & A & A \\
\hline Wc & A & A & A & $A+D$ & $A+D$ \\
\hline \multicolumn{6}{|l|}{ Apple vinegar } \\
\hline W & A & A & A & A & A \\
\hline Wc & A & A & A & $A+D$ & D \\
\hline
\end{tabular}

$W=$ without cap, $W c=$ with cap, $A=a$ life head lice, $D=$ dead head lice, $A+D=a$ life more than dead, $a+D=$ very few alive.

bad level with rate of $20.4 \%$, the lowest rate was among families of good level with rate of 14.35 .

The effect of the natural plant oils were intensified when used with head cap. All plant oils were not effective at 


\begin{tabular}{lllllll} 
Table 5: Some chemical components effect on head lice \\
\hline Chemical components & \multicolumn{5}{c}{$\begin{array}{l}\text { Days of washing or treating } \\
\text { and combing the hair }\end{array}$} \\
\cline { 2 - 7 } & $\mathbf{1}$ & $\mathbf{2}$ & $\mathbf{3}$ & $\mathbf{4}$ & $\mathbf{5}$ \\
\hline Petrol & A & A+D & A+D & D & D \\
W & A+D & D & D & D & D \\
Wc, 2-3h & & & & & \\
Anti- louse shampoos & & & & & \\
Wm & A+D & A+D & A+D & A+D & D \\
Sali & A+D & A+D & A+D & D & D \\
Lice therapy & A+D & A+D & A+D & A+D & D \\
Lycid & & & & & \\
Ordinary hair shampoo (2-3 h. each & & & & & \\
time) & & A & A & A & A & A \\
W & A+D & D & D & D & D \\
Wc &
\end{tabular}

$W=$ without cap, $W c=$ with cap, $W m=$ with comb, $A=a$ life head lice, $D=$ dead head lice, $A+D=a$ life more than dead, $a+D=$ very few a life, $h=$ hour

the maximum time used (48 hour), when used without cap. The most effective was olive and anise, followed by thyme and sesame they were effective at 48 hours after usage. No or very little effect had resulted with garlic oil treatment. Also low effect was recorded with apple vinegar. (Nearly all of natural products treated persons had recurrence infection after two to eight weeks post treatment, except olive and anise).

Petrol was effective at killing the lice, with or without head cap especially after repeating treatment. The anti-lose shampoos were effective after repeating treatment for four to five days. The ordinary hair shampoos had no effect in killing the lice when used solely, however their effect were very greatly intensified when used with head cap. Their effect were appeared after 2-3 hours of usage. Repeating this process for two to three days had completely removed the lice. Fig. 4 shows the dead removed lice after hair shampoos usage as appeared with naked eye. (All of chemical treated persons had no recurrence infection after two weeks to one month post treatment). The insect adult had the ability to still alive on beddings and clothes for two days in very sunny Summer day, but they were die after one day when put in a small glass bottle with strong tide cover.

\section{DISCUSSION}

Pediculosis is remain a public health problem in our country as in some others. In our province the incidence of the infection among children under the study was $14.2 \%$. Females were significantly more (23.6\%)affected than males (3.2\%). The majority

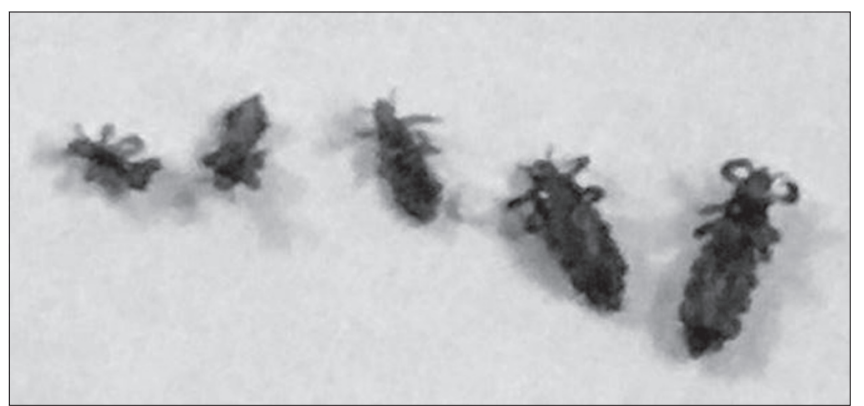

Figure 4: The dead removed lice stages as appeared with naked eye.r.

of the studies had showed identical results. A study in Kirkuk, indicated a prevalence rate of $20 \%$. And girls were infested more $(27.29 \%)$ than boys $(0 \%)$ [3]. An overall rate of infestation was $13.5 \%$ in a Baghdad, and the incidence was significantly higher in girls (17.33\%)than in boys (8.75\%) [6]. In similar study in Jeddah, Saudi, among 5150 school girls examined, HL infection rate was $11.26 \%$ [7]. Also HL infestation rate was $23.32 \%$, the infestation rate was higher in girls (47.12\%) than in boys (0\%). [8]. A report of Centers for Disease Control (CDC) indicated that 6 to 12 million people each year will experience head lice infection in the United States. And that girls have an increased risk rate of infection than boys [14]. Despite the huge development in human life styles and high educational levels of populations, HL is still prevalent. This may be in one hand due that, most of parents are employers or workers and spend a lot of times outdoors. On the other hand, they may be careless and not periodically checking their children heads. In addition to that the direct contact among children in crowded areas as schools and day cars may amplify the chance of transmission. However was the prevalence of HL, females always have head lice infection more than males $[3,6,8]$. This may possibly because, the long hair in females offers good humidity and breeding environment for the insect. A confirmatory datum from a study indicated that, length of the hair is of a very high significant effect on the infection rate. The study found highest rate (22.2\%) in long haired, compared to $10.7 \%$ in medium and $0 \%$ in short haired girls [6]. In current study the most age group which was significantly more infected in both females and males was 7-8 and 9-10 years old, with a rate of 41 , $32.8 \%$ for female and a rate of $13,7 \%$ for males in each age groups respectively. A study found that the age group 3-10 years were most infested with head lice [14]. A lowest infestation rate (12.62\%) was found in 12 years old while the highest $(30.13 \%$ ) 
was in 8 years old [8]. High incidence (6.01-7.61\%) of head lice was delimited in age 6-9 years [3]. A highest rate $(18.7 \%)$ of infection was recorded in age group of $>8-10$ years [6]. This age limitation is probably because of that, the child in this age start to have a type of dependency for bathing and taking care of their cleaning. In such age they may not be able to clean their heads or bodies in a proper way. And this is why the infection rates is lower in smaller or bigger ages in all communities $[3,6,14,15]$. Furthermore, in this age the contact between children will be more, because of playing groups and attending daycares or schools. In the present study, continuous infection significantly was more $(65 \%)$ among girls than in boys. The long of the hair had significantly related with the infestation rate of infection. The highest (22.2\%) was for long haired compared to $10.7 \%$ for medium and $0 \%$ for short haired [6]. This result may be due that, boys in school age in our community will not allowed to have long hair cut. This will aid in noticing the infection easily, and treating it quickly by shaving the hair as done in this area. Also in the current investigation, HL infection had well related with head or body itching. A rate of $44.9 \%$ of infected persons had suffered from head itching and a rate of $15.3 \%$ had suffered from both head and body itching, while $40 \%$ of them had no head or body itching. Similarly, other studies indicated that head itching occur in some persons while some others never experience it $[9,16,17]$. The itching sensation in sensitized person is most likely due to, the saliva secreted form the insect before feeding process or due to the insect soil or other substances. These substances can led to head or localized irritation and erythema. Moreover, however head lice cannot transmit diseases to human but it can cause scalp pyoderma resulted from mechanically transmitted Streptococci and Staphylococci found on the skin, scratching may also cause impetigo or other skin infection, which can resulted in local adenopathy $[15,18]$. Delayed itching sensation to 4-6 weeks post infection may miss early identification and delay treatment process [19,20]. In contrast to the results of most of the studies, that showed significant effect of occupation and educational status $[6,7]$. In current study, the occupation and educational status of the parents were significantly not associated with the frequency of the infection. All families with different levels of economic status had infested child, but the greatest percentage was for intermediate level with rate of $66.5 \%$, followed by bad level with rate of $20.4 \%$. The lowest rate was among families of good level with rate of $14.35 \%$. Most of the studies were agreed with this result $[3,6,7]$. This may because poorer families or families with low outcome may not have good life style. The effect of the natural plant oils in this study were intensified when used with head cap, especially the olive and the anise oils. Anti-lice shampoos were effected without head cap. The anise, ylang-ylang, coconut oils, and isopropyl alcohol were effective at least as permethrin when used in Frankowski et al. (2010)study [9] Several other products have been studied such as andiroba oil, quassia vinegar, melaleuca oil [tea tree oil], lavender oil)[2]. Some home remedies were used in a study, like vinegar, isopropyl alcohol, olive oil, mayonnaise, melted butter and petroleum jelly, tub margarine and thick hair gel. None of these substances were effective in eradicating head lice comparing to insecticides $[6,7,15]$. In Israel some other natural products (coconut oil, anise oil and ylang ylang oil)were as efficacious as the pediculicide. But a vast number of investigators were focused on using of chemical products (insecticide)for head lice treatment $[2,8,9,15,16]$. The effect of the chemicals is because of their toxic effect on the lice that may affect human health too. The petrol is very good and effective but its toxic and flammable. The natural oils action may be because of their ability to exclude air openings in the insect, which led to anoxia and death. But they need repainting washing with a liberal amount of ordinary shampoos to remove them from the hair. In addition to that their toxic effect are not approved yet. Treating infected head with the ordinary hair shampoos with a plastic hair cap is the most recommended, its safe, available and not toxic or flammable.

\section{CONCLUSION}

The petrol is very good and effective but its toxic and flammable. The natural oils action may be because of their ability to exclude air openings in the insect, which led to anoxia and death. But they need repainting washing with a liberal amount of ordinary shampoos to remove them from the hair. In addition to that their toxic effect are not approved yet. Treating infected head with the ordinary hair shampoos with a plastic hair cap is the most recommended, its safe, available and not toxic or flammable. 


\section{REFERENCES}

1. Marquardt WC, Demaree RS and Grieve RB. Parasitology and vector biology. second edition, Academic Press. San Diego, California. 2000; P. 553-556.

2. Devore Cynthia D, MD, FAAP, Schutze Gordon E and MD FAAP., Head Lice. from the American academy of pediatrics. Pediatrics. 2015; 135(5): 1355-1365.

3. Kadir MA., Dawood AS., Al-Sheikhly KT. Prevalence study of head lice among pupils in some primary schools in Kirkuk city and its effects in some of the hematological and biochemical parameters. Tikrit J. Pure Science. 2012; 17(3):1-12.

4. Heukelbach J, Wilcke T, Winter B, et al. Epidemiology and morbidity of scabies and pediculosis capitis in resource-poor communities in Brazil. Br J Dermatol. 2005;153(1):150 -156.

5. Casey $\mathrm{M}$ and Phelan $\mathrm{A}$. Introducing bug busting and action research study to treat and prevent head lice. UCD, Dublin. 2008; 1-101.

6. Mahmood SA. Head pediculosis among in Baghdad area elementary schoolchildren. Iraqi Journal of Science. 2010; 51(1): 49-55.

7. Al-Zanbagi Najia A and Al-Hashdi Dina F. Socio-Economic Status Criteria of Head Lice Prevalence in Jeddah, Saudi Arabia. Acta Parasitologica Globalis.2015;6 (3): 238-245.

8. Rassami Watcharawit and Soonwera Mayura. Epidemiology of pediculosis capitis among schoolchildren in the eastern area of Bangkok, Thailand. Asian Pac J Trop Biomed. 2012; 2(11): 901-904.

9. Frankowski Barbara L, MD, MPH and Bocchini Joseph A. Clinical Report-Head Lice. American Academy of Pediatrics. Pediatrics 2010;126:392-403.

10. Jones KN, English JC $3^{\text {rd }}$. Review of common therapeutic options in the US for the treatment of pediculosis capitis. Clin Infect Dis.2003; 36(11):1355-1361.

11. Meinking TL, Villar ME, Vicaria M, et al. The clinical trials supporting benzyl alcohol lotion 5\% (Ulesfia): a safe and effective topical treatment for head lice (pediculosis humanus capitis). Pediatr Dermatol. 2010; 27(1):19 -24.

12. Greive Kerryn A, Altman Phillip M, Rowe James S, Staton John and JaneV M. A randomised, double-blind, comparative efficacy trial of three head lice treatment options: malathion, pyrethrins with piperonyl butoxide and MOOV Head Lice Solution. Pharmacist.2007; 26 (9)738-743.

13. Frankowski Barbara L and Bocchini Joseph A. Guidance for the Clinician in Rendering Pediatric Care. Clinical Report-Head Lice. American Academy of Pediatrics. Pediatrics. 2010; 126(2): 392-403.

14. Mazurek CM and Lee NP. Medicine Cabinet. How to manage head lice. West J Med. 2000;172:342-345. The Stafford Group. Head lice: evidence-based guidelines based on the Stafford report. 2012 update. Produced by Public Health Medicine Environmental Group, The Stafford Document. On behalf of the Public Health Medicine Environment Group.

15. Finlay J and MacDonald NE. Head lice infestations: A clinical update. Canadian Paediatric Society (CPS)Infectious Diseases and Immunization Committee. Paediatr Child Health. 2008;13(8):692-696. Posted: Oct 12008 Reaffirmed: Feb 12016.

16. Pollack RJ, Kiszewski A and Spielman A. Over diagnosis and consequent mismanagement of head louse infestations in North America. Paediatric Infectious Disease Journal.2000; 19, 689-693.

17. Counihan ML, Andrews RM, Weld H and Speare R.What parents in Australia know and do about head lice. Rural and Remote Health Journal. 2007. www.rrh.org.au.

18. Frankowski BL and Weiner LB. Head Lice. Pediatrics.2002; 110: 638643.

19. Monsen KA and Olson Keller L. A population-based approach to pediculosis management. Public Health Nursing. 2002; 19 (3): 201-208.

20. Mumcuoglu KY, Miller J, Zamir C, Zentner G, Helbin V, Ingber A. The in vivo pediculocidal efficacy of a natural remedy.Isr Med Assoc J. 2002;4:790-793.

Copyright by Obaid HM. This is an open-access article distributed under the terms of the Creative Commons Attribution License, which permits unrestricted use, distribution, and reproduction in any medium, provided the original author and source are credited.

Source of Support: Nil, Conflict of Interest: None declared. 